\section{Cureus}

\title{
Avoiding Toxicity: Insulin Placement in the Portal Vein
}

\author{
Edward D. Murphy ${ }^{1}$, David J. Winchester ${ }^{1}$ \\ 1. NorthShore University Health System, University of Chicago Pritzker School of Medicine
}

$\square$ Corresponding author: Edward D. Murphy, edmurphy77@sbcglobal.net

Disclosures can be found in Additional Information at the end of the article

\section{Abstract}

Peripheral hyperinsulinemia following routine subcutaneous insulin injection in the treated diabetic has multiple morbid sequellae - hypoglycemia, atherosclerosis, heart disease, altered lipid metabolism, hypertension, and impaired hemostasis. We compared portal and peripheral insulin levels following portal or subcutaneous insulin injection in diabetic animals. In nondiabetic animals, we found that portal insulin levels exceed peripheral insulin levels in $95 \%$ of measurements postprandially. In our diabetic animals given insulin subcutaneously, the reverse is true - portal insulin levels exceed peripheral insulin levels in only $8 \%$ of measurements. We ameliorated this peripheral hyperinsulinemia in the treated diabetic animal by accessing a branch of the portal vein for insulin administration: in this way, we found that portal insulin exceeds peripheral insulin in $81 \%$ of our measured postprandial insulin values, more nearly approximating insulin release in the non-diabetic animal.

Categories: Endocrinology/Diabetes/Metabolism, Internal Medicine

Keywords: peripheral hyperinsulinemia, portal, insulin placement, portal vein, insulin toxicity

\section{Introduction}

Peripheral hyperinsuinemia and portal hypoinsulinemia are undesirable but inevitable results following administration of subcutaneous insulin. We studied peripheral and portal insulin levels and ratios following two routes of insulin administration in the diabetic animal during meal tolerance tests. Regular insulin was given subcutaneously or via the portal vein in diabetic, pancreatectomized dogs.

We emphasize our findings using direct insulin injection via catheter into a branch of the portal vein. We compare peripheral and portal insulin levels achieved in this manner with levels achieved after injecting insulin subcutaneously in the diabetic, insulin-deprived dog.

Received 01/24/2013

Review began 01/25/2013

Published 03/12/2013

\section{๑) Copyright 2013}

Murphy et al. This is an open access article distributed under the terms of the Creative Commons Attribution License CC-BY 3.0., which permits unrestricted use, distribution, and reproduction in any medium, provided the original author and source are credited.

\section{Materials And Methods}

Adult healthy mongrel dogs of 18-30 kg were pancreatectomized under general anesthesia with simultaneous placement of catheters in branches of the portal vein and a peripheral vein. Twelve dogs were used, eight diabetic and four control animals. Total pancreatectomy was confirmed by subsequent elevation of fasting and random blood glucose levels into the diabetic range. The portal vein injection catheter tip was placed in a distal branch of the vein. The portal vein sampling catheter tip was placed in the vein close to the liver. The peripheral vein catheter tip was placed in an extremity. All catheters were tunneled subcutaneously to an external pump placed along the dorsal spine, with heparin injection of the catheters then maintained. The external pump was a standard commercial design (Auto Syringe M odd AS*2C) adequate to 


\section{Cureus}

maintain patency with low flow maintenance saline and heparin infusion as well as appropriate insulin infusion at time of meal testing. Our protocol was approved by our organization's animal review committee and institutional review board. Pancreatic enzyme replacement with powdered lipase, protease and amylase was given, adjusted for animal weight. Diabetes was confirmed by increase in blood glucose levels daily after surgery. Healthy animals were studied, diabetic and control. Meal tolerance tests were performed after overnight fasting. Diabetic study groups had similar elevations of fasting blood glucoses and low fasting insulin levels, while receiving intermediate acting insulin twice daily until the day of meal testing.

We measured portal and peripheral glucose and insulin levels before and during four to five hours time following a quickly eaten balanced meal of 625 grams (50\% carbohydrate, $20 \%$ protein, $30 \%$ fat), with regular insulin injection $(0.3 \mathrm{u} / \mathrm{kg})$ given peripherally or portally with this meal. Diabetic study groups on days separate from meal study days were given intermediate acting insulin in dosage adjusted to avoid nocturnal and morning hypoglycemia resulting in elevated fasting glucoses (>140 mg/di) on study days. Insulin levels were measured by standard RIA methods. Glucose assays were performed by glucose oxidase assay.

\section{Results}

In control non-diabetic animals, postprandial portal insulin levels exceeded peripheral insulin levels in 95\% of measurements (Table 1). Similarly, in diabetic animals given insulin via a branch of the portal vein, postprandial portal insulin levels exceeded peripheral insulin levels in $81 \%$ of measurements. However, in diabetic animals given insulin subcutaneously, portal insulin levels exceeded peripheral levels in only $8 \%$ of instances. Subcutaneous insulin administration caused statistically higher peripheral insulin levels and lower portal insulin levels than found in control animals (Table 1).

\begin{tabular}{|c|c|c|c|c|}
\hline & $\begin{array}{l}\text { Peripheral } \\
\text { Insulin (pU/m1) }\end{array}$ & $\begin{array}{l}\text { Portal Insulin } \\
\text { (pU/ml) }\end{array}$ & $\mathbf{N}^{*}$ & $\begin{array}{l}\text { Postprandial Measurements - Por } \\
\text { Insulin Exceeds Peripheral }\end{array}$ \\
\hline Control animals & 23.5 & 83 & 40 & $95 \%$ \\
\hline $\begin{array}{l}\text { Diabetic animals - portal vein } \\
\text { insulin/injection }\end{array}$ & 41 & 46 & 68 & $81 \%$ \\
\hline $\begin{array}{l}\text { Diabetic animals - } \\
\text { subcutaneous insulin/injection }\end{array}$ & $57^{\star \star x}$ & $47.5^{\star}$ & 114 & $8 \%$ \\
\hline
\end{tabular}

${ }^{*} \mathrm{~N}=$ Number of postprandial insulin values in multiple meal ${ }^{* *} \mathrm{p}=$ tolerance tests $<.01$ compared to control animals, Wilkoxon Sum Test. Table I illustrates that portal insulin exceeds peripheral insulin in control animals and portal insulin administered diabetic animals, but not in subcutaneous (SC) insulin injected diabetic animals.

Figure 1 demonstrates mean peripheral to portal insulin ratios in three groups - control animals and diabetic animals given insulin by portal vein or subcutaneously. The overall mean insulin ratios differ among the three groups $(\mathrm{p}<.0001)$. 


\section{Cureus}

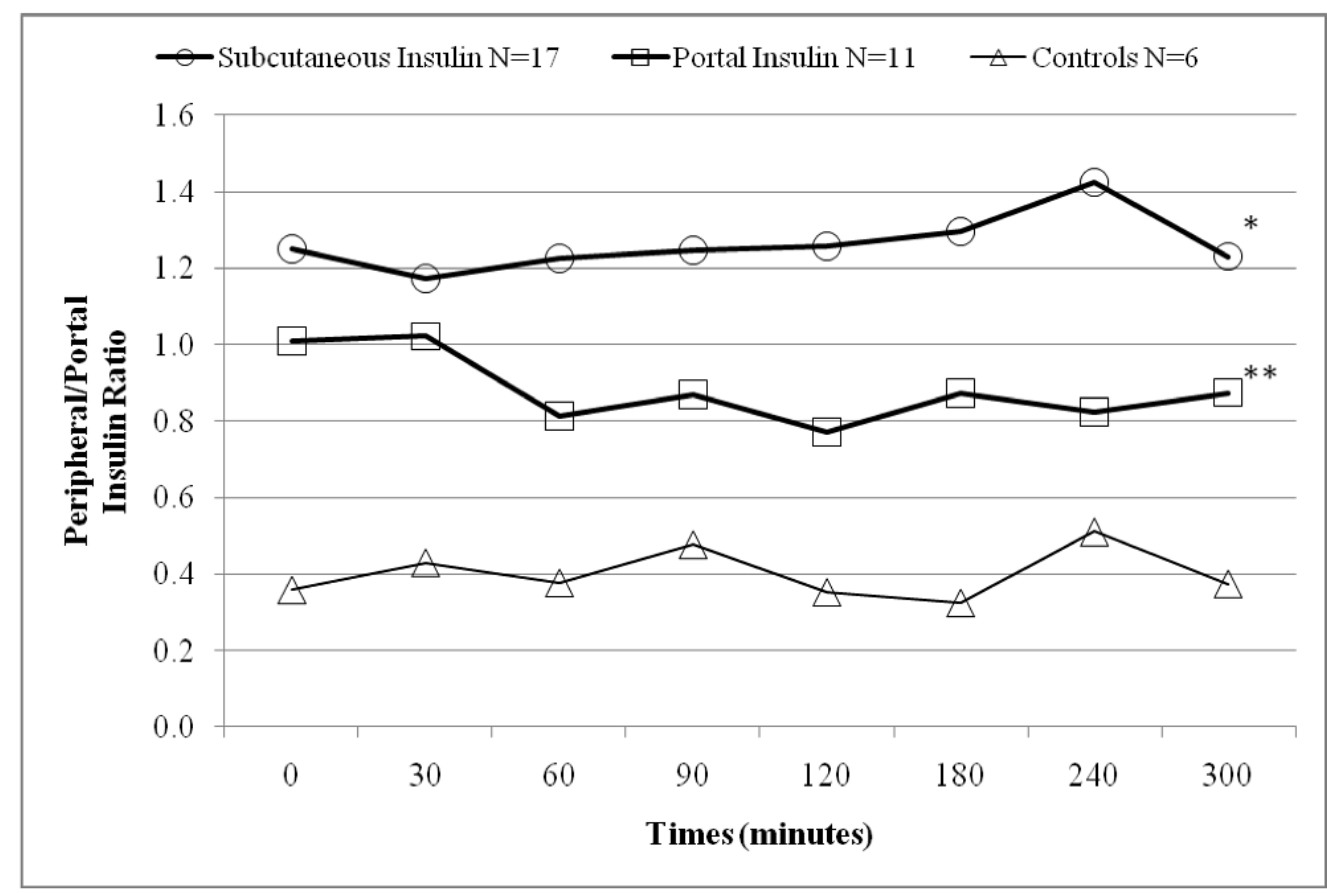

FIGURE 1: Observed mean of peripheral/portal insulin ratio by group fasting or following injection with a meal

\section{Discussion}

In an effort to increase insulin in the portal system and to decrease insulin in the peripheral system, we have evaluated multiple approaches to placement of insulin. In a prior series of preliminary experiments, we administered regular insulin into a cutaneous isolated small bowel segment with portal vein drainage in diabetic dogs [1]. Diabetic control was erratically obtained in this fashion for two weeks. Necessity of surgical procedure to isolate a bowel segment cutaneously with risk of infection or loss of circulation to the bowel segment and uncertainty of precise insulin placement in bowel muscularis led us to abandon insulin placement in the bowel wall.

Injection of insulin into the free peritoneal cavity with absorption into both the portal and peripheral systems has been done in humans [2-4]. This approach using a subcutaneous "button" reservoir for injection and release of insulin has succeeded in establishing glucose control and stabilizing diminished renal function in a group of patients, but fibrosis of catheter tip and steatotic changes at liver surface [5] due to altered triglyceride metabolism have limited use of intraperitoneal insulin placement. As well, HDL cholesterol is decreased and LDL cholesterol and total cholesterol increased in these patients.

Our object principally was to administer insulin subcutaneously and via the portal vein in diabetic animals, achieving a reduction in elevated fasting and postprandial glucose levels to near normal values, while avoiding the peripheral hyperinsulinemia typically noted after subcutaneous insulin injection. On meal test days, we lowered glucoses from an average fasting of $276 \mathrm{mg} / \mathrm{dl}$ to an average nadir of $76 \mathrm{mg} / \mathrm{dl}$ with no statistical differences in glucose control in our diabetic animal groups. We succeeded in establishing portal insulin levels higher than peripheral insulin levels in $81 \%$ of postprandial meal times measured in diabetic animals given insulin via a portal vein branch but in only $8 \%$ of measurements in subcutaneous insulin 
administered instances (Table 1).

Portal insulin administration resulted in peripheral hyperinsulinemia in our diabetic animals (Table 1) compared to controls, though to a lesser extent than subcutaneous administered insulin. A statistical difference in insulin ratios at 60 to 180 minutes between portal and subcutaneous insulin administration levels was achieved (Figure 1), though it remains to be seen if absolute peripheral hyperinsulinemia can be avoided with portal insulin administration. We suggest the ultimate goal of our studies and of management of the insulin dependent diabetic is not only peripheral glucose control but also peripheral and portal "euinsulinemia". We have shown in these studies that insulin administration into the portal vein rather than peripherally subcutaneously begin to more closely approximate control values in the portal vein and the peripheral vein (Table 1) (Figure 1). Probably because we did not maintain "tight" diabetic control routinely in our animals, peripheral/portal insulin ratios (Figure 1) remained higher than that of control animals when we administered portal insulin. More study of the physiologic portal route of insulin administration is needed in seeking to validate this approach.

To emphasize: Peripheral hyperinsulinemia in diabetic patients causes multiple sequellae. Our object here is to avoid peripheral hyperinsulinemia with its morbid sequellae of hypoglycemia, atherosclerosis [6-7], heart disease [8], altered lipid metabolism, hypertension [9], and impaired hemostasis [10]. Achieving peripheral euinsulinemia in diabetic patients is our goal when portal insulin is given in adequate amounts to control diabetes. More than $50 \%$ of this amount will be taken up by the liver in first pass, resulting in more physiologic glucose metabolism [1112] and lowering the risk of peripheral hyperinsulinemia.

Over nearly a century since the isolation and purification of insulin, justifiable pride in its lifesaving use by patients with insulin-dependent diabetes has occurred. The last two decades of insulin pump usage and newer insulin variants have fine-tuned peripheral insulin dosages and glucose management.

Even so, the classic diabetic triad of complications persists - retinopathy, neuropathy and nephropathy. These, along with arterial vessel disease, continue to complicate and shorten the lives of diabetic patients. Peripheral hyperinsulinemia created by subcutaneous placement of insulin, in addition to portal hypoinsulinemia due to lack of pancreatic insulin production and release, remain a non-physiologic pairing. In obtaining a goal, life-saving restoration of physiologic and biologic function by administration of peripheral insulin, we have precipitated an unforeseen result - early vascular disease, morbidity and mortality.

In 1970, the physiologic significance of the secretion of endogenous insulin into the portal circulation was noted [12]. In the presence of a functioning endocrine pancreas portal insulin increases markedly with food absorption after eating, suppressing hepatic glucose production and promoting ingested glucose deposition in the liver [13]. However, the diabetic patient, given insulin subcutaneously, sustains only a subphysiologic modest portal insulin increase, while being placed in a state of peripheral hyperinsulinemia as occurred in our animal model. Consequently, in the insulin-dependent diabetic patient using insulin subcutaneously, the liver does not physiologically suppress hepatic glucose production nor does the liver deposit ingested glucose adequately. Perhaps more importantly, the periphery is exposed to a state of hyperinsulinemia.

Peripheral hyperinsulinemia is associated not only with increased risk of hypoglycemia, but also an increase in catechol and cortisol secretion and lipolysis [14], deleterious effects on vessel walls [6], ischemic heart disease, hypertension and hyperlipidemia [8], and abnormalities in hemostasis [10]. 
Under physiologic circumstances, the concentration of insulin in the portal vein is three to 10 times greater than that in peripheral blood [12], which is approximately what we found in our control animals. Additionally, portal insulin administration indirectly reduces peripheral hyperinsulinemia, as noted by investigations over the last half century. Peripheral hypoglycemia is reduced with portal insulin administration [11-12]. A significant amount of insulin administered via the portal route is extracted by the liver in the first pass, with a lesser portal insulin dosage required to lower peripheral glucose [14-15], resulting in lower peripheral insulin levels, even as portal and peripheral glucose levels are no higher after intra-portal than after subcutaneous insulin placement

Consequently, if intra-portal insulin can be administered in the diabetic patient, the possibility that long-term vascular, lipid or hemostatic abnormalities could be decreased should be anticipated and evaluated.

Pancreas transplantation or islet cell infusion inadequately answers this need. The promise of insulin replacement in the Type I diabetic patients by means of pancreas transplantation or infusion of pancreatic B cells has been only modestly fulfilled in recent years due to limit of transplant organs. In 2009, Khan and Harlan [16] noted that whole pancreas transplantation was performed in about 2,000 patients in the U.S. in the preceding year and that about 6,000 pancreatic B cell contents were available for islet cell placement into recipient cell liver sinusoids via the portal venous system. Of these latter 6,000 donors, about two were needed for every one recipient. Consequently, about 5,000 (2,000 transplants and 3,000 islet cell recipients) Type I diabetic patients in the U.S. received functioning islet cells in 2008. Insulin independence is achieved in only a minority of islet cell transplanted patients [17]. Presently the majority of islet cell transplants patients continue to receive peripheral insulin subcutaneous injection. The estimated pool of Type I diabetic patients in the U.S. in 2009 was $5 \%$ of 23.6 million diabetics, or 1.18 million patients [17]. Thus, islet cell or pancreas transplanted patients amounted to about $0.4 \%$ of Type I patients in a year. This obviously leaves $99.6 \%$ of Type I patients in need of more physiologic insulin placement and replacement than is currently done.

Additionally, pancreas transplantation particularly with its need for immunosuppression has not always increased survival in transplant recipients [18]. However, a number of clues suggest that insulin placed in the portal venous system is less-causative of hypoglycemia $[11,12,15,19]$, may lead to less local vessel stenosis in the infused location and better vessel neointimal growth after injury [20], and may avoid peripheral hyperinsulinemia. So a catheter, a stent or a device access placed in a branch of the portal system, available for exogenous insulin injection or infusion, would better mimic non-diabetic insulin release and delivery to the liver before reaching the periphery.

\section{Conclusions}

With portal vein insulin administration, we obtained higher portal insulin levels than peripheral insulin levels in our animal model resulting in more nearly physiologic "euinsulinemia". The promise of a resultant potential decrease in ischemic heart disease, lipid abnormalities, abnormalities in hemostasis, and less frequent, less severe hypoglycemia lend urgency to development of a means to place insulin into the portal venous system. Appropriate "euinsulinemia", both portally and peripherally, should be achievable by direct administration into a branch of the portal system, whether via umbilical vein or a more deeply placed stent or catheter in the portal system.

\section{Additional Information}

\section{Disclosures}


Human subjects: All authors have confirmed that this study did not involve human participants or tissue. Animal subjects: The NorthShore University Health System Issued protocol number N/A. Conflicts of interest: In compliance with the ICMJE uniform disclosure form, all authors declare the following: Payment/services info: This work was entirely supported by patient donations to the Endocrine Research Fund of Evanston Hospital, NorthShore University HealthSystem. Financial relationships: All authors have declared that they have no financial relationships at present or within the previous three years with any organizations that might have an interest in the submitted work. Other relationships: The protocol was approved by The NorthShore University Health System's animal review committee and institutional review board.

\section{Acknowledgements}

Dr. E. Dennis Murphy is the guarantor of this work, had full access to all the data, and takes full responsibility for the integrity of data and the accuracy of data analysis. Acknowledgments: The authors would like to thank Hongyan Du MB, MS, PSTAT ${ }^{\circledR}$ for her statistical assistance as well as Baikun Wang and Patricia Kallio for technical assistance. Peter C. Butler MD and John Phair MD kindly reviewed our manuscript. This work was entirely supported by patient donations to the Endocrine Research Fund of Evanston Hospital, NorthShore University HealthSystem.

\section{References}

1. Murphy ED, Buzard KA, Scanlon EF: Portal Vein Insulin Placement in Cutaneous Bowel Wall Injection, Abstract. American Diabetes Association Annual Meeting. 1980.

2. Schade DS, Eaton RP: The peritoneum - a potential insulin delivery route for a mechanical pancreas. Diabetes Care. 1980, 3:229-34.

3. Lahtel a JT, Mustonen J, Pasternack A: Comparison of intraperitoneal and subcutaneous insulin administration on insulin sensitivity and serum lipids in type I diabetic patients on continuous ambulatory peritoneal dialysis treatment. Clin Sci (Lond). 1995, 88:427-32.

4. Radzuik J, Pye S, Seigler DE, Skyler JS, Offord R, Davies G: Splanchnic and systemic absorption of intraperitoneal insulin using a new double-tracer method. Am J Physiol. 1994, 226(5 Pt1):E750-9.

5. Wanless IR, Bargman JM, Orepoulos DG, Vas SI: Subcapsular steEttonecrosis in response to peritoneal insulin delivery: a clue to the pathogenesis of steatonecrosis obesity. Modern Pathol. 1989, 2:69-74.

6. Sobel BE: Potentiation of vasculopathy by insulin: Implications from an NHLBI clinical alert. Circulation. 1996, 93:1613-15.

7. Stolar MW: Atherosclerosis in diabetes: The role of hyperinsulinemia. Metabolism. 1988, 37(2 Suppl 1):1-9.

8. Despres JP, Lamarche B, Mauriege P, Cantin B, Dagenais GR, Moorjani S, Lupien PJ: Hyperinsulinemia as an independent risk factor for ischemic heart disease . NEJM. 1996, 334:952-7.

9. Salonen JT, Lakka TA, Lakka HM, Valkonen VP, Everson SA, Kaplan GA: Hyperinsulinemia is associated with the incidence of hypertension and dyslipidemia in middle-aged men. Diabetes. 1998, 47:270-5.

10. Meigs JB, Mittleman MA, Nathan DM, Tofler GH, Singer DE, Murphy-Sheehy PM, Lipinska I, D'Agostino RB, Wilson PW: Hyperinsulinemia, hyperglycemia and impaired homeostasis: The Framingham Offspring Study. JAMA. 2000, 283:221-8.

11. Felig P: Insulin rates and routes of delivery. NEJM. 1974, 291:1031-2.

12. Blackard WG, Nelson NC: Portal and peripheral immunoreactive insulin concentrations before and after glucose infusion. Diabetes. 1970, 19:302-6.

13. Sindelar DK, Chu CA, Venson P, Donahue EP, Neal DW, Cherrington AD: Basal hepatic glucose production is regulated by the portal vein insulin concentration. Diabetes. 1998, 47:523-9.

14. Davis SN, Goldstein RE, Jacobs J, Price L. Wolfe R, Cherrington AD: The effects of differing insulin levels on the hormonal and metabolic response to equivalent hypoglycemia in normal humans. Diabetes. 1993, 42:263-72. 


\section{Cureus}

15. Madison LL, Unger RH: The physiologic significance of the secretion of endogenous insulin into the portal circulation. I. Comparison of the effects of glucagon-free insulin administered via the portal vein and via a peripheral vein on the magnitude of hypoglycemia and peripheral glucose utilization. J Clin Invest. 1958, 37:631=9.

16. Khan MH, Harlan DM: Counterpoint: clinical isle transplantation: not ready for primetime . Diabetes Care. 2009, 32:1570-4.

17. American Diabetic Association 70th Annual Meeting Orlando, Florida Introduction . (2010). Accessed: 2011: http://professional.diabetes.org/CongressReport_Display.aspx?CID=81727.

18. Venstrom JM, McBride MA, Rother K I, Hirshberg B, Orchard TJ, Harland DM: Survival after pancreas transplantation in patients with diabetes and preserved kidney function. JAMA. 2003, 290:2817-23.

19. Newell KA, Bruce DS, Cronin DC, Woodle ES, Millis JM , Piper JB, Huss E, Thistlethwaite JR Jr: Comparison of pancreas transplantation with portal venous and enteric exocrine drainage to the standard technique utilizing bladder drainage of exocrine secretions. Transplantation. 1996, 62:1353-6.

20. Breen DM, Chan KK, Dhaliwall JK, Ward MR, Al Koudsi N, Lam L, De Souza M, Ghanim H, Dandona P, Stewart DJ, Bendeck M P, Giacca A: Insulin increases reendothelialization and inhibits cell migration and neointimal growth after arterial injury. Arterioscl Throm Basc Biol. 2009, 29:1060-6. 